\title{
Dấu ấn KHXHNV Việt trên các tạp chí quốc tế có hệ số tác động cao năm 2018
}

\author{
Trần Trung \\ Học viện Dân tộc \\ Vũ Thị Hạnh \\ Trường Đại học Ngoại thương \\ Hoàng Phương Hạnh \\ Viện Khoa học Giáo dục Việt Nam
}

Ngày 20-06-2019 vừa qua, Clarivate Analytics đã công bố hệ số tác động (HSTĐ) Journal Impact Factor (JIF) 2018 thông qua báo cáo Journal Citation Reports (JCR) 2019. Báo cáo JCR 2019 năm nay có tổng số 11.877 tạp chí, trong đó, có 283 tạp chí mới và 138 tạp chí cho phép truy cập miễn phí. 64\% số tạp chí có JIF tăng so với báo cáo năm 2018.

Bài viết này trình bày một số thống kê về khoa học xã hội nhân văn Việt Nam (KHXHNV) dựa trên dữ liệu JIF mới được công bố. Đầu tiên, cần khẳng định rằng việc đánh giá chất lượng của các nghiên cứu khoa học chỉ dựa trên JIF và Citescore là không phù hợp. Hệ số tác động như JIF và CiteScore được xây dựng dựa trên giả định rằng một tạp chí khoa học sẽ có uy tín cao, phạm vi tác động lớn khi các bài báo xuất bản ở đó được trích dẫn nhiều lần trong các bài báo khác của cùng hoặc khác tạp chí, vì vậy không hoàn toàn phản ánh chất lượng. Vì thế, thống kê trong bài viết này chỉ được đưa ra trên cơ sở cung cấp thông tin tham khảo.

Có thể dễ dàng nhận thấy một tỷ trọng lớn các CBQT tập trung vào các ấn phẩm có mức JIF<3. Tuy nhiên, khoảng JIF 3-5 cũng vẫn cho thấy con số tương đối các nghiên cứu của Việt Nam. Từ mức JIF $\geq 5$, số lượng các công bố giảm, chiếm tỷ trọng rất nhỏ trong tổng số các ấn phẩm có JIF. 
Hình 1. Phân bố tổng thể các công bố quốc tế trên các tạp chí ISI đã có JIF năm 2018

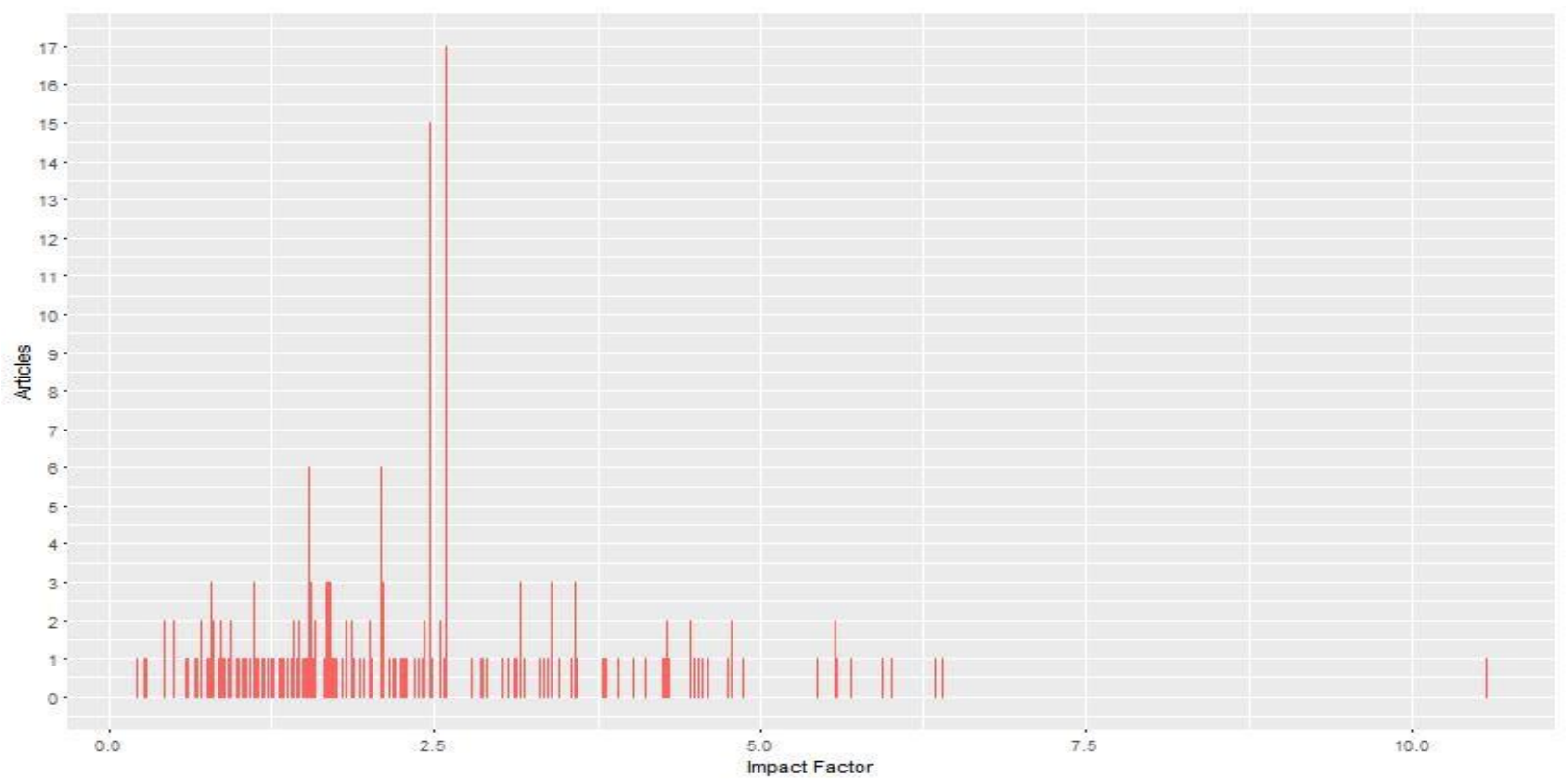

\section{Cơ quan Việt Nam có nghiên cứu với JIF cao trong năm 2018}

Bảng 1 dưới đây thống kê các cơ quan tại Việt Nam có công bố trong lĩnh vực KHXHNV năm 2018 trên các tạp chí có $\mathrm{JIF} \geq 5$. Nhìn chung, hầu hết các cơ quan trong nước có năng lực nghiên cứu mạnh đều tập trung vào các ấn phẩm có mức JIF tốt nhất trong khoảng 5-6.

Trong năm 2018, đã có một công bố của Trung tâm Nghiên cứu Xã hội Liên ngành - ISR, trường Đại học Phenikaa - trên tạp chí có JIF 2 chữ số: 10.575. Bên cạnh đó, ISR cũng có các nghiên cứu khác với JIF lần lượt bằng 5.688 và 5.929 . Ngay sau đó là Khoa Ngân hàng - Tài chính, trường Đại học Ngoại thương với JIF = 7.724.

Đồng thời số liệu năm nay cũng cho thấy sự xuất hiện khá ấn tượng của Khoa Kinh tế, Đại học Tây Nguyên với bài trên tạp chí có JIF 6.395. Trong dữ liệu cung cấp ở Bảng 1, còn có các cơ quan lớn như ĐHQG Hà Nội và ĐHQG Thành phố Hồ Chí Minh, hai trong số các đơn vị hàng đầu về nghiên cứu tại Việt Nam hiện nay.

Bảng 1. Các cơ quan Việt Nam có nghiên cứu KHXHNV trong năm 2018 trên ấn phẩm có $\mathrm{JIF} \geq 5^{*}$

\begin{tabular}{|l|l|}
\hline Cơ quan & JIF \\
\hline Centre for Interdisciplinary Social Research - Phenikaa University & 10.575 \\
\hline Faculty of Banking and Finance - Foreign Trade University & 7.724 \\
\hline Faculty of Economics - Tay Nguyen University & 6.395 \\
\hline Centre for Interdisciplinary Social Research - Phenikaa University & 5.929 \\
\hline Institute of Philosophy - Vietnam Academy of Social Sciences & 5.929 \\
\hline Phu Xuan University & 5.929 \\
\hline
\end{tabular}




\begin{tabular}{|l|l|}
\hline Vietnam Panorama Media Monitoring & 5.929 \\
\hline Centre for Interdisciplinary Social Research - Phenikaa University & 5.688 \\
\hline Institute for Social Development Studies & 5.688 \\
\hline School of Law - Vietnam National University Hanoi & 5.688 \\
\hline Ho Chi Minh City University of Agriculture and Forestry & 5.589 \\
\hline Can Tho University & 5.572 \\
\hline College of Environment and Natural Resources & 5.572 \\
\hline Hanoi University of Science and Technology & 5.572 \\
\hline Vietnam National University Ho Chi Minh City & 5.572 \\
\hline RMIT University Vietnam & 5.439 \\
\hline
\end{tabular}

( *Ghi chú: dũ liệu do các tác giả thu thập, do đó có thể không hoàn toàn đầy đủ)

\section{Một số bài CBQT của tác giả Việt trên ấn phẩm có JIF cao nhất trong năm 2018}

Bảng 2 trình bày 25 nghiên cứu KHXHNV trên các tạp chí có JIF trong khoảng từ 4.253 đến 10.575. Bài quan điểm về chi phí khoa học, đầu tư và làm khoa học hiệu quả tại các nền kinh tế đang phát triển được TS. Vương Quân Hoàng (ISR, trường Đại học Phenikaa, Hà Nội) công bố trên Nature Human Behaviour có JIF cao nhất trong năm 2018, ở mức 10.575. Ngoài ra, đại diện Trung tâm ISR còn có 2 CBQT khác trên các tạp chí Scientific Data [5.929] và Journal of Clinical Medicine [5.688]. Tiêu biểu về mức năng suất trên ấn phẩm JIF cao là tác giả Nguyễn Trung Thành (Leibniz University Hannover) với 5 CBQT. Nhà nghiên cứu trẻ Hồ Hữu Lộc thuộc ĐHQG Thành phố Hồ Chí Minh dẫn dắt tới 3 CBQT trên các ấn phẩm mạnh là Ecosystem Services [Elsevier; JIF 5.572] và Ecological Indicators [JIF 4.49].

Xét theo từng ngành, có khá nhiều sản phẩm khoa học được đăng trên các tạp chí thuộc lĩnh vực môi trường/bền vững (11). Lĩnh vực quản trị, bao gồm cả quản trị kinh doanh, du lịch, lẫn môi trường cũng có 8 nghiên cứu. Lĩnh vực y xã hội có các công bố trên Journal of Clinical Medicine [5.688], International Journal of Drug Policy [4.528], JMIR mHealth and uHealth [4.301]. Ngành kinh tế cũng có các bài viết chất lượng trên các tạp chí Journal of International Business Studies [7.724], Journal of Business Venturing [6.395], Journal of Peasant Studies [4.754], Ecological Economics [4.281] và Land Degradation and Development [4.275]. Sự phát triển của khoa học xã hội, thể hiện qua các công bố quốc tế, hiện nay dựa rất nhiều vào năng suất của ngành kinh tế. Tuy nhiên, nghiên cứu khoa học trong ngành kinh tế có sự cạnh tranh rất lớn và để có thể bước chân vào các tạp chí với JIF cao trong lĩnh vực nàsy không phải là một nhiệm vụ dễ dàng.

Bảng 2. 25 CBQT của các tác giả KHXHNV Việt Nam trong năm 2018 và JIF tương ứng

\begin{tabular}{|l|l|l|l|l|}
\hline Tên nghiên cứu & Ref & Tác giả & Ấn phẩm & JIF \\
\hline $\begin{array}{l}\text { The (ir)rational consideration of the } \\
\text { cost of science in transition } \\
\text { economies }\end{array}$ & {$[1]$} & $\begin{array}{l}\text { Vuong Quan } \\
\text { Hoang }\end{array}$ & $\begin{array}{l}\text { Nature Human } \\
\text { Behaviour }\end{array}$ & 10.575 \\
\hline $\begin{array}{l}\text { Policy uncertainty, derivatives use, } \\
\text { and firm-level FDI }\end{array}$ & {$[2]$} & $\begin{array}{l}\text { Quang Nguyen; } \\
\text { Kim Huong Trang; }\end{array}$ & $\begin{array}{l}\text { Journal of } \\
\text { International } \\
\text { Business Studies }\end{array}$ & 7.724 \\
\hline
\end{tabular}




\begin{tabular}{|c|c|c|c|c|}
\hline & & \begin{tabular}{|l|} 
Marina \\
Papanastassiou
\end{tabular} & & \\
\hline $\begin{array}{l}\text { Eco-efficiency analysis of } \\
\text { sustainability-certified coffee } \\
\text { production in Vietnam }\end{array}$ & [3] & $\begin{array}{l}\text { Ho Quoc Thong; } \\
\text { Hoang Viet Ngu; } \\
\text { Clevo Wilson; } \\
\text { Nguyen Trung } \\
\text { Thanh }\end{array}$ & $\begin{array}{l}\text { Journal of } \\
\text { Cleaner } \\
\text { Production }\end{array}$ & 6.395 \\
\hline $\begin{array}{l}\text { Institutional quality and market } \\
\text { selection in the transition to market } \\
\text { economy }\end{array}$ & [4] & Tran Thu Hien & $\begin{array}{l}\text { Journal of } \\
\text { Business } \\
\text { Venturing }\end{array}$ & 6.333 \\
\hline $\begin{array}{l}\text { Scenario-based planning for } \\
\text { tourism development using system } \\
\text { dynamic modelling: A case study } \\
\text { of Cat Ba Island, Vietnam }\end{array}$ & [5] & $\begin{array}{l}\text { Thanh Mai; Carl } \\
\text { Smith }\end{array}$ & $\begin{array}{l}\text { Tourism } \\
\text { Management }\end{array}$ & 6.012 \\
\hline $\begin{array}{l}\text { An open database of productivity in } \\
\text { Vietnam's social sciences and } \\
\text { humanities for public use }\end{array}$ & [6] & $\begin{array}{l}\text { Vuong Quan } \\
\text { Hoang; La Viet } \\
\text { Phuong; Vuong } \\
\text { Thu Trang; Ho } \\
\text { Manh Toan; } \\
\text { Nguyen To Hong } \\
\text { Kong; Nguyen To } \\
\text { Viet Ha; Pham } \\
\text { Hung Hiep; Ho } \\
\text { Manh Tung }\end{array}$ & Scientific Data & 5.929 \\
\hline $\begin{array}{l}\text { The Dark Side of Female HIV } \\
\text { Patient Care: Sexual and } \\
\text { Reproductive Health Risks in Pre- } \\
\text { and Post-Clinical Treatments }\end{array}$ & [7] & $\begin{array}{l}\text { Khuat Thu Hong; } \\
\text { Do Thu Trang; } \\
\text { Nguyen Thi Van } \\
\text { Anh; Vu Xuan } \\
\text { Thai; Nguyen Thi } \\
\text { Phuong Thao; Tran } \\
\text { Kien; Ho Manh } \\
\text { Tung; Nguyen To } \\
\text { Hong Kong; } \\
\text { Vuong Thu Trang; } \\
\text { La Viet Phuong; } \\
\text { Vuong Quan } \\
\text { Hoang }\end{array}$ & \begin{tabular}{|l|} 
Journal of \\
Clinical Medicine
\end{tabular} & 5.688 \\
\hline $\begin{array}{l}\text { Decreased use of pesticides for } \\
\text { increased yields of rice and fish- } \\
\text { options for sustainable food } \\
\text { production in the Mekong Delta }\end{array}$ & [8] & $\begin{array}{l}\text { Hakan Berg; } \\
\text { Nguyen Thanh } \\
\text { Tam }\end{array}$ & $\begin{array}{l}\text { Science of the } \\
\text { Total } \\
\text { Environment }\end{array}$ & 5.589 \\
\hline $\begin{array}{l}\text { The legal aspects of Ecosystem } \\
\text { Services in agricultural land } \\
\text { pricing, some implications from a } \\
\text { case study in Vietnam's Mekong } \\
\text { Delta }\end{array}$ & [9] & $\begin{array}{l}\text { Ho Huu Loc; } \\
\text { Irvine Kim Neil; } \\
\text { Nguyen Thi Hong } \\
\text { Diep; Nguyen Thi } \\
\text { Kim Quyen; }\end{array}$ & $\begin{array}{l}\text { Ecosystem } \\
\text { Services }\end{array}$ & 5.572 \\
\hline
\end{tabular}




\begin{tabular}{|c|c|c|c|c|}
\hline & & $\begin{array}{l}\text { Nguyen Ngoc Tue; } \\
\text { Yoshihisa Shimizu }\end{array}$ & & \\
\hline $\begin{array}{l}\text { Socio-geographic indicators to } \\
\text { evaluate landscape Cultural } \\
\text { Ecosystem Services: A case of } \\
\text { Mekong Delta, Vietnam }\end{array}$ & {$[10]$} & $\begin{array}{l}\text { Ho Huu Loc; } \\
\text { Thomas J. } \\
\text { Ballatore; Irvine } \\
\text { Kim Neil; Nguyen } \\
\text { Thi Hong Diep; } \\
\text { Truong Thi Cam } \\
\text { Tien; Yoshihisa } \\
\text { Shimizu }\end{array}$ & $\begin{array}{l}\text { Ecosystem } \\
\text { Services }\end{array}$ & 5.572 \\
\hline $\begin{array}{l}\text { Constraints to the uptake of solar } \\
\text { home systems in Ho Chi Minh City } \\
\text { and some proposals for } \\
\text { improvement }\end{array}$ & [11] & $\begin{array}{l}\text { Bob Baulch; Do } \\
\text { Thuy Duong; Le } \\
\text { Thai Ha }\end{array}$ & $\begin{array}{l}\text { Renewable } \\
\text { Energy }\end{array}$ & 5.439 \\
\hline $\begin{array}{l}\text { Questioning triple rice } \\
\text { intensification on the Vietnamese } \\
\text { mekong delta floodplains: An } \\
\text { environmental and economic } \\
\text { analysis of current land-use trends } \\
\text { and alternatives }\end{array}$ & [12] & $\begin{array}{l}\text { Tran Duc Dung; } \\
\text { Gerardo van } \\
\text { Halsema; Petra } \\
\text { J.G.J. Hellegers; } \\
\text { Fulco Ludwig; } \\
\text { Andrew Wyatt }\end{array}$ & $\begin{array}{l}\text { Journal of } \\
\text { Environmental } \\
\text { Management }\end{array}$ & 4.865 \\
\hline $\begin{array}{l}\text { Cross-functional knowledge } \\
\text { sharing, coordination and firm } \\
\text { performance: The role of cross- } \\
\text { functional competition }\end{array}$ & [13] & $\begin{array}{l}\text { Nguyen Phong } \\
\text { Nguyen; Ngo Viet } \\
\text { Liem; Tania Bucic; } \\
\text { Nguyen Dong } \\
\text { Phong }\end{array}$ & $\begin{array}{l}\text { Industrial } \\
\text { Marketing } \\
\text { Management }\end{array}$ & 4.779 \\
\hline $\begin{array}{l}\text { Value synergy and value } \\
\text { asymmetry in relationship } \\
\text { marketing programs }\end{array}$ & [14] & $\begin{array}{l}\text { Ngoc Luu; Ngo } \\
\text { Viet Liem; Jack } \\
\text { Cadeaux }\end{array}$ & $\begin{array}{l}\text { Industrial } \\
\text { Marketing } \\
\text { Management }\end{array}$ & 4.779 \\
\hline $\begin{array}{l}\text { Flex crops or flex livelihoods? The } \\
\text { story of a volatile commodity chain } \\
\text { in upland northern Vietnam }\end{array}$ & [15] & $\begin{array}{l}\text { Sarah Turner; } \\
\text { Annuska Derks; } \\
\text { Ngo Thuy Hanh }\end{array}$ & $\begin{array}{l}\text { Journal of } \\
\text { Peasant Studies }\end{array}$ & 4.754 \\
\hline $\begin{array}{l}\text { Realising the value of knowledge } \\
\text { resources and capabilities: an } \\
\text { empirical study }\end{array}$ & [16] & $\begin{array}{l}\text { Nguyen Thi } \\
\text { Nguyet Que; Ngo } \\
\text { Viet Liem; } \\
\text { Gavin Northey; } \\
\text { Christopher } \\
\text { Agyapong Siaw }\end{array}$ & $\begin{array}{l}\text { Journal of } \\
\text { Knowledge } \\
\text { Management }\end{array}$ & 4.604 \\
\hline $\begin{array}{l}\text { Assessing resource-use efficiency } \\
\text { of land use }\end{array}$ & [17] & $\begin{array}{l}\text { Alexander Herzig; } \\
\text { Nguyen Trung } \\
\text { Thanh; Anne- } \\
\text { Gaelle E. Ausseil; } \\
\text { Ganga Ram } \\
\text { Maharjan; John R. }\end{array}$ & $\begin{array}{l}\text { Environmental } \\
\text { Modelling and } \\
\text { Software }\end{array}$ & 4.552 \\
\hline
\end{tabular}




\begin{tabular}{|c|c|c|c|c|}
\hline & & $\begin{array}{l}\text { Dymond; } \\
\text { Sebastian Arnhold; } \\
\text { Thomas Koellner; } \\
\text { Daniel Rutledge; } \\
\text { John Tenhunen }\end{array}$ & & \\
\hline $\begin{array}{l}\text { Health-related work productivity } \\
\text { loss is low for patients in a } \\
\text { methadone maintenance program in } \\
\text { Vietnam }\end{array}$ & [18] & $\begin{array}{l}\text { Tran Xuan Bach; } \\
\text { Nguyen Hoang } \\
\text { Long; Nguyen Tat } \\
\text { Cuong; Carl A. } \\
\text { Latkin }\end{array}$ & $\begin{array}{l}\text { International } \\
\text { Journal of Drug } \\
\text { Policy }\end{array}$ & 4.528 \\
\hline $\begin{array}{l}\text { An analytical approach in } \\
\text { accounting for social values of } \\
\text { ecosystem services in a Ramsar } \\
\text { site: A case study in the Mekong } \\
\text { Delta, Vietnam }\end{array}$ & [19] & $\begin{array}{l}\text { Ho Huu Loc; } \\
\text { Nguyen Thi Hong } \\
\text { Diep; Vo Thanh } \\
\text { Tuan; Yoshihisa } \\
\text { Shimizu }\end{array}$ & $\begin{array}{l}\text { Ecological } \\
\text { Indicators }\end{array}$ & 4.49 \\
\hline $\begin{array}{l}\text { Distribution channels and } \\
\text { ownership of upmarket hotels in a } \\
\text { transition economy }\end{array}$ & [20] & $\begin{array}{l}\text { Le Ngoc Tuan; } \\
\text { Douglas G. Pearce; } \\
\text { Karen A. Smith }\end{array}$ & $\begin{array}{l}\text { International } \\
\text { Journal of } \\
\text { Hospitality } \\
\text { Management } \\
\end{array}$ & 4.465 \\
\hline $\begin{array}{l}\text { E-satisfaction and continuance } \\
\text { intention: The moderator role of } \\
\text { online ratings }\end{array}$ & [21] & $\begin{array}{l}\text { Tran Trong Thuy; } \\
\text { Pham Thi Minh } \\
\text { Ly; Le Tuan Loc }\end{array}$ & $\begin{array}{l}\text { International } \\
\text { Journal of } \\
\text { Hospitality } \\
\text { Management } \\
\end{array}$ & 4.465 \\
\hline $\begin{array}{l}\text { What drives young Vietnamese to } \\
\text { use mobile-health innovations? } \\
\text { Implications for health } \\
\text { communication and behavioral } \\
\text { interventions }\end{array}$ & {$[22]$} & $\begin{array}{l}\text { Tran Xuan Bach; } \\
\text { Melvyn W. B. } \\
\text { Zhang; Le Thi } \\
\text { Huong; Nguyen } \\
\text { Duc Hinh; Nguyen } \\
\text { Hoang Long; } \\
\text { Nguyen Thi Le } \\
\text { Quyen; Tran Dinh } \\
\text { Tho; Carl A. } \\
\text { Latkin; Roger } \\
\text { Chun Man Ho }\end{array}$ & $\begin{array}{l}\text { JMIR mHealth } \\
\text { and uHealth }\end{array}$ & 4.301 \\
\hline $\begin{array}{l}\text { Are Ecosystem Services } \\
\text { Complementary or Competitive? } \\
\text { An Econometric Analysis of Cost } \\
\text { Functions of Private Forests in } \\
\text { Vietnam }\end{array}$ & [23] & $\begin{array}{l}\text { Cosmas Kombat } \\
\text { Lambini; Nguyen } \\
\text { Trung Thanh; Jens } \\
\text { Abildtrup; Pham } \\
\text { Van Dien; John } \\
\text { Tenhunen; Serge } \\
\text { Garcia }\end{array}$ & $\begin{array}{l}\text { Ecological } \\
\text { Economics }\end{array}$ & 4.281 \\
\hline $\begin{array}{l}\text { Is Environmental Income Reporting } \\
\text { Evasive in Household Surveys? } \\
\text { Evidence From Rural Poor in Laos }\end{array}$ & {$[24]$} & $\begin{array}{l}\text { Priyanka Parvathi; } \\
\text { Nguyen Trung } \\
\text { Thanh }\end{array}$ & $\begin{array}{l}\text { Ecological } \\
\text { Economics }\end{array}$ & 4.281 \\
\hline
\end{tabular}




\begin{tabular}{|l|l|l|l|l|}
\hline $\begin{array}{l}\text { Natural resource extraction and } \\
\text { household welfare in rural Laos }\end{array}$ & {$[25]$} & $\begin{array}{l}\text { Nguyen Trung } \\
\text { Thanh; Do Truong } \\
\text { Lam; Ulrike Grote }\end{array}$ & $\begin{array}{l}\text { Land Degradation } \\
\text { and Development }\end{array}$ & 4.275 \\
\hline $\begin{array}{l}\text { Buyer-supplier exchange } \\
\text { relationship: How do exchange } \\
\text { partners behave across the } \\
\text { relationship life-cycle? }\end{array}$ & {$[26]$} & $\begin{array}{l}\text { Booi Kam; Lai } \\
\text { Manh Khang }\end{array}$ & $\begin{array}{l}\text { Transportation } \\
\text { Research Part E: } \\
\text { Logistics and } \\
\text { Transportation } \\
\text { Review }\end{array}$ & 4.253 \\
\hline
\end{tabular}

(*Ghi chú: dũ liệu do các tác giả thu thập, do đó có thể không hoàn toàn đầy đủ)

Nghiên cứu khoa học tại Việt Nam nói chung và KHXHNV nói riêng đang trong giai đoạn đầu hội nhập với thế giới. Vấn đề tiếp cận với các tiêu chuẩn quốc tế về xuất bản các sản phẩm khoa học đã được Nhà nước cũng như các cơ quan nghiên cứu quan tâm. Điều này được thể hiện qua các quy định, tiêu chí về đánh giá và xếp loại chất lượng công việc cũng như xét duyệt học vị và chức danh trong lĩnh vực học thuật và nghiên cứu khoa học. Trong đó, bên cạnh các quy định về số lượng công bố khoa học trên các tạp chí quốc tế, một số yêu cầu cụ thể về chất lượng cũng đã bước đầu được đưa vào quy định như chỉ mục và xếp hạng của tạp chí [27]. Thống kê mang tính chất tham khảo về JIF của các công bố KHXHNV tại Việt Nam cho thấy các tín hiệu khả quan, mặc dù còn trong giai đoạn sơ khởi, về sự quan tâm đối với các yếu tố quốc tế về chất lượng trong nghiên cứu khoa học của Việt Nam.

Note: Bài viết được đăng trên tạp chí Khoa học \& Phát triển: http://khoahocphattrien.vn/khoa-hoc/dau-an-khxhnv-viet-nam-tren-cac-tap-chi-quoc-te-co-he-sotac-dong-cao-nam-2018/20190624113145746p1c160.htm; và SciComm SSHPA: https://sc.sshpa.com/post/5507.

\section{References:}

[1] Vuong, Q. H. (2018). The (ir) rational consideration of the cost of science in transition economies. Nature Human Behaviour, 2, 5, DOI: 10.1038/s41562-017-0281-4.

[2] Nguyen, Q., Kim, T., \& Papanastassiou, M. (2018). Policy uncertainty, derivatives use, and firm-level FDI. Journal of International Business Studies, 49(1), 96-126, DOI: 10.1057/s41267-017-0090-z.

[3] Ho, T. Q., Hoang, V. N., Wilson, C., \& Nguyen, T. T. (2018). Eco-efficiency analysis of sustainability-certified coffee production in Vietnam. Journal of Cleaner Production, 183, 251-260, DOI: 10.1016/j.jclepro.2018.02.147.

[4] Tran, H. T. (2018). Institutional quality and market selection in the transition to market economy. Journal of Business Venturing, DOI: 10.1016/j.jbusvent.2018.07.001.

[5] Mai, T., \& Smith, C. (2018). Scenario-based planning for tourism development using system dynamic modelling: A case study of Cat Ba Island, Vietnam. Tourism Management, 68, 336-354, DOI: 10.1016/j.tourman.2018.04.005. 
[6] Vuong, Q. H., La, V. P., Vuong, T. T., Ho, M. T., Nguyen, H. K. T., Nguyen, V. H., ... \& Ho, M. T. (2018). An open database of productivity in Vietnam's social sciences and humanities for public use. Scientific Data, 5. 180188, DOI: 10.1038/sdata.2018.188.

[7] Khuat, T., Do, T., Nguyen, V., Vu, X., Nguyen, P., Tran, K., ... \& Vuong, Q. (2018). The dark side of female HIV patient care: Sexual and reproductive health risks in pre-and postclinical treatments. Journal of Clinical Medicine, 7(11), 402, DOI: 10.3390/jcm7110402.

[8] Berg, H., \& Tam, N. T. (2018). Decreased use of pesticides for increased yields of rice and fish-options for sustainable food production in the Mekong Delta. Science of the Total Environment, 619, 319-327, DOI: 10.1016/j.scitotenv.2017.11.062.

[9] Loc, H. H., Irvine, K. N., Diep, N. T. H., Quyen, N. T. K., Tue, N. N., \& Shimizu, Y. (2018). The legal aspects of Ecosystem Services in agricultural land pricing, some implications from a case study in Vietnam's Mekong Delta. Ecosystem Services, 29, 360-369, DOI: 10.1016/j.ecoser.2016.11.019.

[10] Loc, H. H., Ballatore, T. J., Irvine, K. N., Nguyen, T. H. D., Truong, T. C. T., \& Yoshihisa, S. (2018). Socio-geographic indicators to evaluate landscape Cultural Ecosystem Services: A case of Mekong Delta, Vietnam. Ecosystem Services, 31, 527-542, DOI: 10.1016/j.ecoser.2017.11.003.

[11] Baulch, B., Do, T. D., \& Le, T. H. (2018). Constraints to the uptake of solar home systems in Ho Chi Minh City and some proposals for improvement. Renewable energy, 118, 245-256, DOI: 10.1016/j.renene.2017.10.106.

[12] Tran, D. D., van Halsema, G., Hellegers, P. J., Ludwig, F., \& Wyatt, A. (2018). Questioning triple rice intensification on the Vietnamese Mekong delta floodplains: an environmental and economic analysis of current land-use trends and alternatives. Journal of Environmental Management, 217, 429-441, DOI: 10.1016/j.jenvman.2018.03.116.

[13] Nguyen, N. P., Ngo, L. V., Bucic, T., \& Phong, N. D. (2018). Cross-functional knowledge sharing, coordination and firm performance: The role of cross-functional competition. Industrial Marketing Management, 71, 123-134, DOI: 10.1016/j.indmarman.2017.12.014.

[14] Luu, N., Ngo, L. V., \& Cadeaux, J. (2018). Value synergy and value asymmetry in relationship marketing programs. Industrial Marketing Management, 68, 165-176, DOI: 10.1016/j.indmarman.2017.10.011.

[15] Turner, S., Derks, A., \& Thúy Hạnh, N. (2019). Flex crops or flex livelihoods? The story of a volatile commodity chain in upland northern Vietnam. Journal of Peasant Studies, 46(2), 276-296, DOI: 10.1080/03066150.2017.1382477.

[16] Nguyen, T. N. Q., Ngo, L. V., Northey, G., \& Siaw, C. A. (2019). Realising the value of knowledge resources and capabilities: an empirical study. Journal of Knowledge Management, 23(2), 374-395, DOI: 10.1108/jkm-09-2016-0372. 
[17] Herzig, A., Nguyen, T. T., Ausseil, A. G. E., Maharjan, G. R., Dymond, J. R., Arnhold, S., ... $\&$ Tenhunen, J. (2018). Assessing resource-use efficiency of land use. Environmental Modelling \& Software, 107, 34-49, DOI: 10.1016/j.envsoft.2018.05.005.

[18] Tran, B. X., Nguyen, L. H., Nguyen, C. T., \& Latkin, C. A. (2018). Health-related work productivity loss is low for patients in a methadone maintenance program in Vietnam. International Journal of Drug Policy, 60, 1-7, DOI: 10.1016/j.drugpo.2018.07.007.

[19] Loc, H. H., Diep, N. T. H., Tuan, V. T., \& Shimizu, Y. (2018). An analytical approach in accounting for social values of ecosystem services in a Ramsar site: A case study in the Mekong Delta, Vietnam. Ecological Indicators, 89, 118-129, DOI: 10.1016/j.ecolind.2017.12.066.

[20] Ngoc, T. L., Pearce, D. G., \& Smith, K. A. (2018). Distribution channels and ownership of upmarket hotels in a transition economy. International Journal of Hospitality Management, 68, 50-58, DOI: 10.1016/j.ijhm.2017.09.008.

[21] Tran, L. T. T., Pham, L. M. T., \& Le, L. T. (2019). E-satisfaction and continuance intention: The moderator role of online ratings. International Journal of Hospitality Management, 77, 311-322, DOI: 10.1016/j.ijhm.2018.07.011.

[22] Tran, B. X., Zhang, M. W., Le, H. T., Nguyen, H. D., Nguyen, L. H., Nguyen, Q. L. T., ... \& Ho, R. C. (2018). What Drives Young Vietnamese to Use Mobile Health Innovations? Implications for Health Communication and Behavioral Interventions. JMIR mHealth and uHealth, 6(11), e194, DOI: 10.2196/mhealth.6490.

[23] Lambini, C. K., Nguyen, T. T., Abildtrup, J., Tenhunen, J., \& Garcia, S. (2018). Are ecosystem services complementary or competitive? An econometric analysis of cost functions of private forests in Vietnam. Ecological Economics, 147, 343-352, DOI: 10.1016/j.ecolecon.2018.01.029.

[24] Parvathi, P., \& Nguyen, T. T. (2018). Is environmental income reporting evasive in household surveys? Evidence from rural poor in Laos. Ecological Economics, 143, 218-226, DOI: 10.1016/j.ecolecon.2017.07.022.

[25] Nguyen, T. T., Do, T. L., \& Grote, U. (2018). Natural resource extraction and household welfare in rural Laos. Land Degradation \& Development, 29(9), 3029-3038, DOI: 10.1002/ldr.3056.

[26] Kam, B. H., \& Lai, M. K. (2018). Buyer-supplier exchange relationship: How do exchange partners behave across the relationship life-cycle?. Transportation Research Part E: Logistics and Transportation Review, 113, 239-257, DOI: 10.1016/j.tre.2017.12.007.

[27] Vuong, Q. H. (2019). Breaking barriers in publishing demands a proactive attitude. Nature Human Behaviour, 3, DOI: 10.1038/s41562-019-0667-6. 
